\title{
Compact dipole nanoantenna coupler to plasmonic slot waveguide.
}

\author{
Andryieuski, Andrei; Malureanu, Radu; Biagi, Giulio; Holmgaard, Tobias; Lavrinenko, Andrei
}

Published in:

Optics Letters

Link to article, DOI:

10.1364/OL.37.001124

Publication date:

2012

Document Version

Publisher's PDF, also known as Version of record

Link back to DTU Orbit

Citation (APA):

Andryieuski, A., Malureanu, R., Biagi, G., Holmgaard, T., \& Lavrinenko, A. (2012). Compact dipole nanoantenna coupler to plasmonic slot waveguide. Optics Letters, 37(6), 1124-1126. https://doi.org/10.1364/OL.37.001124

\section{General rights}

Copyright and moral rights for the publications made accessible in the public portal are retained by the authors and/or other copyright owners and it is a condition of accessing publications that users recognise and abide by the legal requirements associated with these rights.

- Users may download and print one copy of any publication from the public portal for the purpose of private study or research.

- You may not further distribute the material or use it for any profit-making activity or commercial gain

- You may freely distribute the URL identifying the publication in the public portal 


\title{
Compact dipole nanoantenna coupler to plasmonic slot waveguide
}

\author{
Andrei Andryieuski, ${ }^{1, *}$ Radu Malureanu, ${ }^{1}$ Giulio Biagi, ${ }^{2}$ Tobias Holmgaard, ${ }^{2}$ and Andrei Lavrinenko ${ }^{1}$ \\ ${ }^{1}$ DTU Fotonik, Technical University of Denmark, Oersteds pl. 343, 2800 Kongens Lyngby, Denmark \\ ${ }^{2}$ Department of Physics and Nanotechnology, Aalborg University, Skjernvej 4A, 9220 Aalborg East, Denmark \\ *Corresponding author: andra@fotonik.dtu.dk
}

Received November 25, 2011; revised January 18, 2012; accepted January 26, 2012;

posted January 27, 2012 (Doc. ID 158936); published March 15, 2012

\begin{abstract}
Optical nanoantennas can be used for coupling radiation to or from waveguides in analogy to micro- and radio-wave systems. In this letter we provide a systematic description of the design approaches for a coupler to a plasmonic slot waveguide in the telecom range around $1.55 \mu \mathrm{m}$ with realistic excitation from a lensed optical fiber. We show that the best coupling efficiency of $26 \%$ can be achieved by utilizing a dipole antenna with side and bottom reflectors, and such coupling efficiency is 185 times larger than for the bare waveguide. The nanoantenna coupler provides a compact interface between an optical fiber and a plasmonic slot waveguide for future optical integrated circuits. () 2012 Optical Society of America

OCIS codes: $250.5403,240.6680,250.5300$.
\end{abstract}

Plasmonic slot waveguides [1] provide both subwavelength mode confinement and propagation length on the order of tens of micrometers. That makes them promising for high-density optical circuits and interconnects [2]. The small size of the slot mode, however, makes it difficult to couple light to it directly from the free space or from an optical fiber.

To overcome this challenge several coupling approaches have been proposed. Delacour et al. [3] suggested using a complex system containing a silicon grating, a tapered waveguide, and a directional coupler from a silicon waveguide to a plasmonic slot waveguide. The measured coupling efficiency of the directional coupler was $70 \%$. Combined with the silicon grating with maximal efficiency of $69 \%$ [4] the total efficiency of the whole system can be as high as $48 \%$. However, a coupler that consists of several parts results in a large device size.

The most compact coupling solution is a plasmonic nanoantenna. Optical antennas have been used for fluorescence enhancement and sensing [5], light-emitting diodes []], lasers [7]], photodiodes []], and solar cells [9], but only recently have nanoantennas been applied to coupling [10-12]. In the abovementioned works [10-12] dipole or bowtie antennas were used. The maximal reported coupling efficiency (CE), which is the ratio of the power launched into the waveguide to the incident power, of the antenna system [11] for telecom wavelength $1.55 \mu \mathrm{m}$ was about $15 \%$. To obtain such CE the light was focused to a small excitation spot with the diameter about $0.9 \mu \mathrm{m}$ (area of $0.64 \mu \mathrm{m}^{2}$ ) with a high numerical aperture (NA) microscope objective (NA $=0.9$ ). Such an excitation scheme is suitable for laboratory experiments, but for practical applications it is desirable to use optical fibers.

In this contribution we provide a systematic approach to design an efficient, broadband, and compact dipole antenna nanocoupler for the telecom wavelength range around $1.55 \mu \mathrm{m}$ in a vertical coupling configuration with a realistic excitation directly from an optical fiber.

The role of the nanoantenna [Fig. 1(a)] is to capture electromagnetic radiation from free space and to launch it into a waveguide. There are several reasons for the power not to be fully launched into the waveguide: (1) Some power can be absorbed due to ohmic losses. (2) The power cannot be fully launched due to impedances mismatch. These issues can, however, be solved by antenna geometry optimization. (3) Some captured power is reradiated to the surrounding medium. The reradiated power can be partially returned with the help of reflectors [see Fig. 1(b)]. In the very best case of a lossless antenna perfectly matched to the waveguide, the CE cannot be larger than $50 \%$ [13]. (4) Some power can pass straight through without being captured due to the spatial mismatch between the excitation spot and antenna

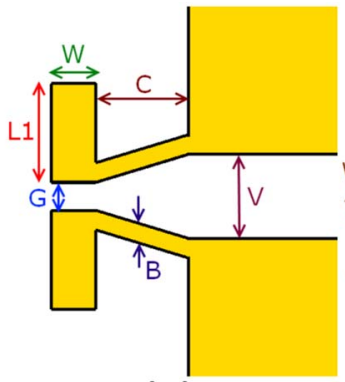

(a)

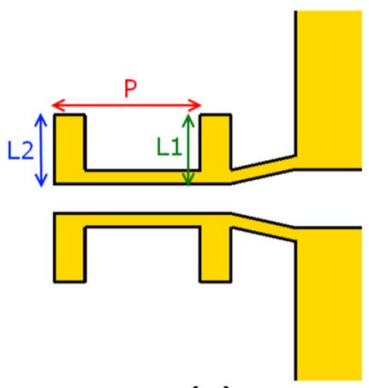

(c)

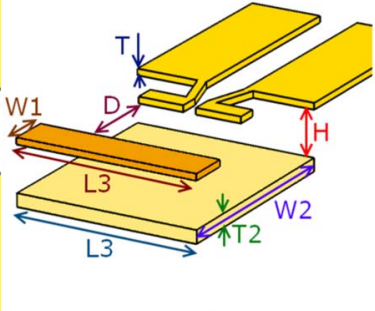

(b)

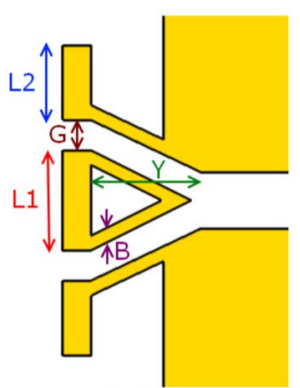

(d)
Fig. 1. (Color online) Nanoantenna coupler design with geometrical parameters marked. (a) Single antenna coupler. (b) Antenna with side and bottom reflectors. (c) Serial and (d) parallel connections of nanoantennas in an array. The parameters of the nanoantennas not specified in (b) through (d) are the same as in (a). Antennas and waveguide are made from gold and embedded in silica (not shown). 
effective area. To increase the $\mathrm{CE}$ one can use several antennas connected in an array. Two configurations are possible: the serial [Fig. 1(c)] and parallel connections [Fig. 1(d)].

We made the simulation and optimization with CST Microwave Studio [14]. The simulation domain size was $10.5 \times 1.1 \times 6 \mu \mathrm{m}^{3}$. The typical time for one simulation with a 12-core processor was $5 \mathrm{~min}$. The nanoantenna and waveguide of thickness $T=50 \mathrm{~nm}$ were made from gold, which was considered as a Drude metal with plasma frequency $\omega_{P}=1.37 \times 10^{16} \mathrm{~s}^{-1}$ and collision frequency $\omega_{C}=8.0 \times 10^{13} \mathrm{~s}^{-1}$ [15]. The metallic structures were embedded in silica (refractive index $n=1.47$ ). The silica substrate below the waveguide was considered as infinite. The silica thickness above the waveguide was $250 \mathrm{~nm}$. The symmetric configuration of the waveguide (silica substrate and superstrate) ensured absence of power leakage to gold-silica surface plasmon polaritons and radiation modes [16]. The excitation source (port 1) was placed in vacuum $300 \mathrm{~nm}$ above the silica surface. The excitation spot had a Gaussian profile with the effective diameter (at the $1 / e^{2}$ intensity level) of $2.5 \mu \mathrm{m}$, which corresponds to the area of $4.9 \mu \mathrm{m}^{2}$. Such a spot can be easily achieved with a commercially available lensed fiber [17].

Some physical considerations on the initial parameter values should be mentioned as well-chosen initial values ensure fast convergence. (1) The dipole antenna works efficiently when its length is proportional to an integer number of half-wavelengths. Even though the plasmon wavelength is different from the wavelength of light in the surrounding dielectric medium, the total initial length of the antenna about $\lambda / 2 n=530 \mathrm{~nm}$ gives a good starting approximation. (2) The distance to the side and bottom reflectors should be such that the waves reflected back to the antenna return in phase. For the bottom reflector this gives $H=\lambda / 4 n=265 \mathrm{~nm}$. Moreover, the incident wave reflected from the bottom mirror forms the maximum of a standing wave at the antenna position, and that improves nanocoupler efficiency. (3) Antennas in the serial array [Fig. 1(c)] should be placed at such distance that the plasmons excited by them interfere constructively; that means the distance $P$ should be equal to the plasmon wavelength.

Optimization was made in two steps. First, we varied geometrical parameters (Fig. 1) one by one, and those that gave the maximal transmission $\left(S_{21}\right)$ parameter were selected as an initial approximation. Then we made numerical optimization with the simplex method provided within the CST Microwave Studio for variation range $\pm 30 \%$ of initial values. The optimized values of parameters are shown in Table 1.

We calculated the $S_{21}$ of the Gaussian beam to the slot mode. The length of the waveguide from the nanoantenna to the detecting port 2 was $L=5 \mu \mathrm{m}$. The CE (Fig. 2) was restored from $S_{21}(\lambda)$ spectra, taking into account plasmon decay upon propagation in the waveguide [propagation length $L_{P}(\lambda)$ ] and reflection at the air-silica interface (reflectivity $R=0.036$ )

$$
\mathrm{CE}(\lambda)=S_{21}^{2}(\lambda) \exp \left[L / L_{P}(\lambda)\right] /(1-R) .
$$

Table 1. Optimized Geometrical Parameters

\begin{tabular}{|c|c|c|c|}
\hline Parameter & Antenna $(a-b)$ & Serial Array (c) & Parallel Array (d) \\
\hline $\mathrm{B}(\mathrm{nm})$ & 80 & 80 & 80 \\
\hline $\mathrm{C}(\mathrm{nm})$ & 370 & 370 & 385 \\
\hline $\mathrm{D}(\mathrm{nm})$ & 570 & - & - \\
\hline $\mathrm{G}(\mathrm{nm})$ & 150 & 150 & 160 \\
\hline H (nm) & 255 & - & - \\
\hline $\mathrm{L} 1$ (nm) & 353 & 295 & 535 \\
\hline $\mathrm{L} 2$ (nm) & - & 415 & 400 \\
\hline L3 (nm) & 3000 & - & - \\
\hline $\mathrm{P}(\mathrm{nm})$ & - & 775 & - \\
\hline $\mathrm{T}(\mathrm{nm})$ & 50 & 50 & 50 \\
\hline $\mathrm{T} 2$ (nm) & 100 & - & - \\
\hline V (nm) & 300 & 300 & 300 \\
\hline W (nm) & 150 & 150 & 150 \\
\hline $\mathrm{W} 1$ (nm) & 1000 & - & - \\
\hline $\mathrm{W} 2$ (nm) & 2500 & - & - \\
\hline $\mathrm{Y}(\mathrm{nm})$ & - & - & 585 \\
\hline
\end{tabular}

The excitation configuration and electric field images at $\lambda=1.55 \mu \mathrm{m}$ are shown in Fig. 3 . The waveguide alone has a very low $\mathrm{CE}=0.14 \%$ [Fig. 2(a)]. Therefore, the excited plasmons are hardly visible in Fig. 3(b). Adding a single nanoantenna, the $\mathrm{CE}$ is increased to $14 \%$ [Figs. 2(a) and 3(c)]. This value is in good correspondence with the previously reported result [11] except the fact that in our case the excitation spot area is 8 times larger. With the bottom and bottom-and-side reflectors the coupling efficiency is $24 \%$ and $26 \%$, respectively [Figs. 2(a) and 3(d)]. This is half of the $50 \%$ theoretical limit due to a large spatial beam profile and ohmic losses. Making the Gaussian spot smaller, we achieved CE up to $40 \%$ (not shown). At the same time such $\mathrm{CE}=26 \%$ is tremendously larger (185 times) than the CE of the bare waveguide. It is remarkable that the bandwidth of the

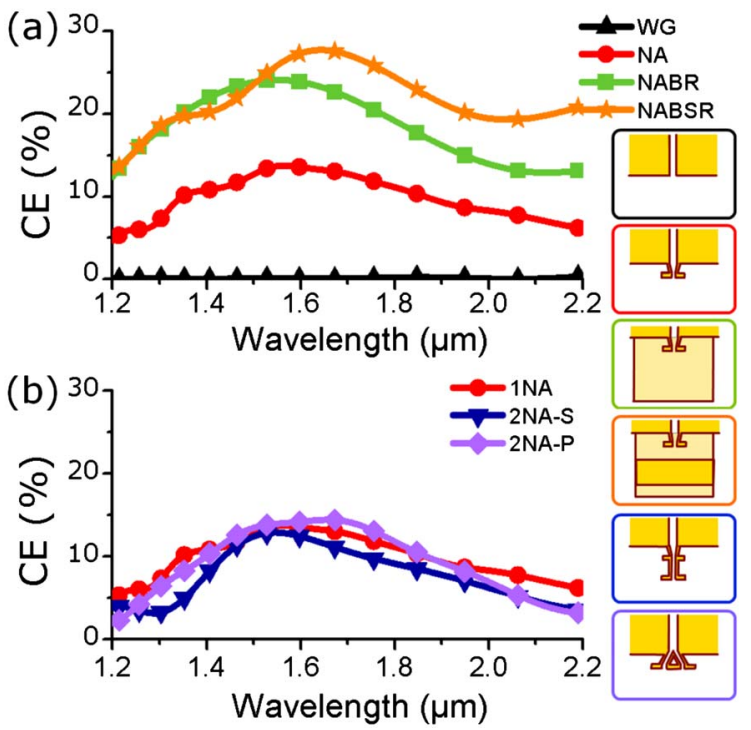

Fig. 2. (Color online) Coupling efficiency spectra of the antenna systems: (a) waveguide without antenna (WG, black triangles up), single nanoantenna (NA, red circles), antenna with bottom (NABR, green squares) and bottom and side reflector (NASBR, orange stars), (b) one antenna (1, red circles) two antennas connected in series ( $2 S$, blue triangles down) and in parallel $(2 P$, violet diamonds). 


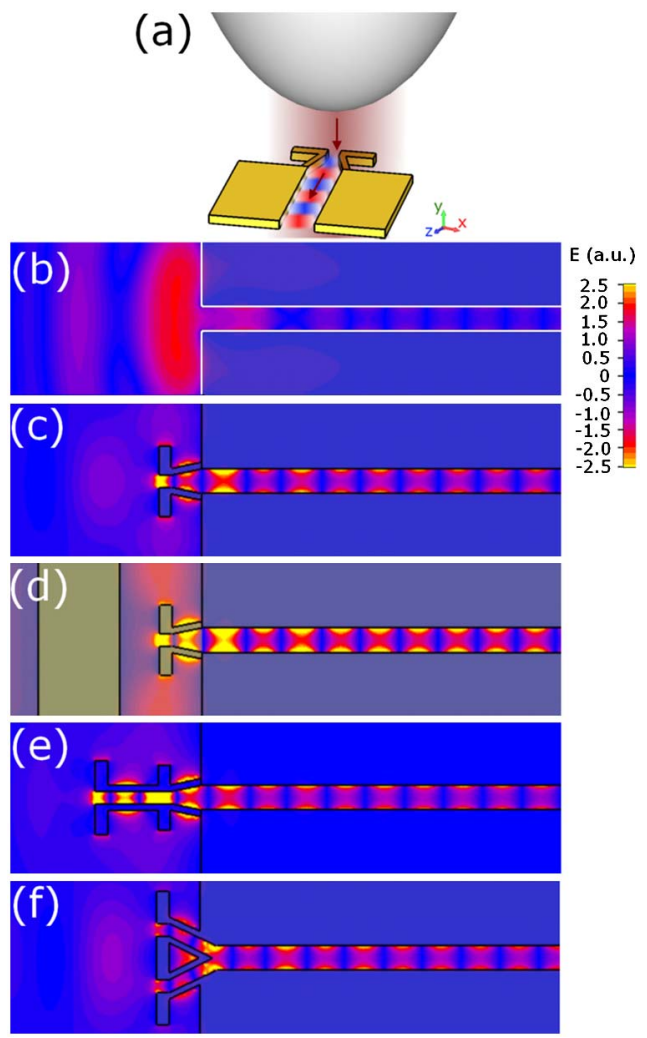

Fig. 3. (Color online) (a) Antenna working in vertical coupling configuration. The beam from the fiber excites slot plasmons. Instant images of the simulated electric field at $\lambda=1.55 \mu \mathrm{m}$ when coupling to: (b) waveguide only, (c) single antenna, (d) antenna with side and bottom reflectors, (e) serial, and (f) parallel antenna array. The color scale is equal for (b) through (f).

nanoantenna coupler is extremely large. It covers the entire telecom range and even more-from $1.22 \mu \mathrm{m}$ to $2.14 \mu \mathrm{m}$. An alignment sensitivity analysis showed that the $\mathrm{CE}$ is larger than 0.5 of the maximal $\mathrm{CE}$ for the fiber tip position within $-0.8 \mu \mathrm{m}<x<0.8 \mu \mathrm{m}, 0.1 \mu \mathrm{m}<y<$ $2.3 \mu \mathrm{m},-0.8 \mu \mathrm{m}<z<0.5 \mu \mathrm{m}(x=0, y=0, z=0$ is the antenna center). Changing the length $L_{1}$ by $10 \mathrm{~nm}$ leads to a negligible CE decrease of $0.3 \%$, which confirms the robustness of the design to fabrication errors.

Comparing the single nanoantenna performance with serial and parallel arrays, we admit that their coupling efficiencies are almost identical [see Figs. 2(b), 3(c), $3(\mathrm{e})$, and 3(f)]. The array of antennas can capture more radiation from a larger area. However, the losses upon delivery of the power to the slot diminish the advantages of the array. In the parallel array [Fig. 3(f)], the Y-coupler combining the waves has a transmittivity of $25 \%$ only. In the serial array the connecting wire has a low propagation length $(2 \mu \mathrm{m})$, and the power captured by the right antenna is not only forwarded to the waveguide but also to the left antenna [Fig. 3(e)]. Moreover, the maximum of the Gaussian beam coincides with the right antenna, so the left one is in a weaker excitation field. However, the conclusion that an array of two antennas gives no CE increase is only true for a specific excitation spot. In the simulations with a plane wave excitation we observed an increase of plasmon intensity with an increasing number of antennas.

An antenna nanocoupler is the most compact solution for the slot waveguide excitation. There are several approaches to increase the efficiency of the nanoantenna coupler: geometry optimization, adding reflectors, and using antenna arrays. We have shown that for the excitation with a lensed optical fiber the best design is the single antenna with side and bottom reflectors. A coupling efficiency of $26 \%$ can be achieved with a conventional lensed fiber. The broad bandwidth and small footprint makes the antenna coupler promising in plasmonic applications.

A. A., R. M., and A. L. acknowledge funding from the Danish Council for Technical and Production Sciences (NIMbus project). G. B. and T. H. acknowledge funding from the Danish Council for Independent Research no. 09-072949.

\section{References}

1. L. Liu, Z. Han, and S. He, Opt. Express 13, 6645 (2005).

2. D. K. Gramotnev and S. I. Bozhevolnyi, Nat. Photon. 4, 83 (2010).

3. C. Delacour, S. Blaize, P. Grosse, J. M. Fedeli, A. Bruyant, R. Salas-Montiel, G. Lerondel, and A. Chelnokov, Nano Lett. 10, 2922 (2010).

4. D. Vermeulen, S. Selvaraja, P. Verheyen, G. Lepage, W. Bogaerts, P. Absil, D. Van Thourhout, and G. Roelkens, Opt. Express 18, 18278 (2010).

5. C. E. Talley, J. B. Jackson, C. Oubre, N. K. Grady, C. W. Hollars, S. M. Lane, T. R. Huser, P. Nordlander, and N. J. Halas, Nano Lett. 5, 1569 (2005).

6. D. Dey, J. Kohoutek, R. M. Gelfand, A. Bonakdar, and H. Mohseni, Opt. Lett. 35, 2783 (2010).

7. C.-Y. Cho, M.-K. Kwon, S.-J. Lee, S.-H. Han, J.-W. Kang, S.-E. Kang, D.-Y. Lee, and S.-J. Park, Nanotechnology 21, 205201 (2010)

8. M. W. Knight, H. Sobhani, P. Nordlander, and N. J. Halas, Science 332, 702 (2011).

9. H. A. Atwater and A. Polman, Nat. Mater. 9, 205 (2010).

10. Z. Fang, L. Fan, C. Lin, D. Zhang, A. J. Meixner, and X. Zhu, Nano Lett. 11, 1676 (2011).

11. J. Wen, P. Banzer, A. Kriesch, D. Ploss, B. Schmauss, and U. Peschel, Appl. Phys. Lett. 98, 101109 (2011).

12. J. Huang, T. Feichtner, P. Biagioni, and B. Hecht, Nano Lett. 9, 1897 (2009).

13. C. Balanis, Antenna Theory: Analysis and Design (WileyInterscience, 2005)

14. CST, "Computer Simulation Technology, AS," http://cst .com.

15. A. D. Rakic, A. B. Djurisic, J. M. Elazar, and M. L. Majewski, Appl. Opt. 37, 5271 (1998).

16. G. Veronis and S. Fan, J. Lightwave Technol. 25, 2511 (2007).

17. OZ Optics Ltd., http://ozoptics.com. 